Draft Version May 25, 2022

Preprint typeset using $\mathrm{IAT}_{\mathrm{E}} \mathrm{X}$ style emulateapj v. 11/26/03

\title{
ASYMPTOTIC SELF-SIMILAR SOLUTIONS WITH A CHARACTERISTIC TIME-SCALE
}

\author{
ELI WAXMan ${ }^{1}$ AND DOv ShVARTs ${ }^{2,3}$
}

(Dated: May 25, 2022)

Draft version May 25, 2022

\begin{abstract}
For a wide variety of initial and boundary conditions, adiabatic one dimensional flows of an ideal gas approach self-similar behavior when the characteristic length scale over which the flow takes place, $R$, diverges or tends to zero. It is commonly assumed that self-similarity is approached since in the $R \rightarrow \infty(0)$ limit the flow becomes independent of any characteristic length or time scales. In this case, the flow fields $f(r, t)$ must be of the form $f(r, t)=t^{\alpha_{f}} F(r / R)$ with $R \propto( \pm t)^{\alpha}$. We show that requiring the asymptotic flow to be independent only of characteristic length scales implies a more general form of self-similar solutions, $f(r, t)=R^{\delta_{f}} F(r / R)$ with $\dot{R} \propto R^{\delta}$, which includes the exponential $(\delta=1)$ solutions, $R \propto e^{t / \tau}$. We demonstrate that the latter, less restrictive, requirement is the physically relevant one by showing that the asymptotic behavior of accelerating blast-waves, driven by the release of energy at the center of a cold gas sphere of initial density $\rho \propto r^{-\omega}$, changes its character at large $\omega$ : The flow is described by $0 \leq \delta<1, R \propto t^{1 /(1-\delta)}$, solutions for $\omega<\omega_{c}$, by $\delta>1$ solutions with $R \propto(-t)^{1 /(\delta-1)}$ diverging at finite time $(t=0)$ for $\omega>\omega_{c}$, and by exponential solutions for $\omega=\omega_{c}$ ( $\omega_{c}$ depends on the adiabatic index of the gas, $\omega_{c} \sim 8$ for $4 / 3<\gamma<5 / 3$ ). The properties of the new solutions obtained here for $\omega \geq \omega_{c}$ are analyzed, and self-similar solutions describing the $t>0$ behavior for $\omega>\omega_{c}$ are also derived.

Subject headings: hydrodynamics - shock waves — supernovae: general
\end{abstract}

\section{INTRODUCTION}

Self-similar solutions to the hydrodynamic equations describing adiabatic one dimensional flows of an ideal gas are of interest for several reasons. The non-linear partial differential hydrodynamic equations are reduced for self-similar flows to ordinary differential equations, which greatly simplifies the mathematical problem of solving the equations and in certain cases allows one to find analytic solutions. Moreover, self-similar solutions often describe the limiting behavior approached asymptotically by flows which take place over a characteristic scale, $R$, which diverges or tends to zero (see Sedov 1959; Zel'Dovich \& Raizer 1967; Barenblatt 1996, for reviews). Some examples of such asymptotic solutions which are widely used in astrophysical contexts are the Sedov-von Neumann-Taylor solutions (Sedov 1946; Von Neumann 1947; Taylor 1950) describing expanding decelerating spherical blast waves, for which $R \rightarrow \infty$, and the Gandel'Man-Frank-Kamenetskii-Sakurai solutions Gandel'Man \& Frank-Kamenetskii 1956; Sakurai 1960) describing the emergence of a shock wave from the surface of a star, for which $R \rightarrow 0$. Both types of solutions are relevant, e.g., to supernova explosions and in particular to the recently detected shock breakouts (e.g. Matzner \& McKee 1999; Waxman et al. 2007). An extensive discussion of spherical self-similar blast-waves in an astrophysical context is given by Ostriker \& McKee (1988).

It is commonly assumed that self-similarity is approached since in the $R \rightarrow \infty(0)$ limit the flow becomes

\footnotetext{
${ }^{1}$ Department of Particle Physics \& Astrophysics, Weizmann Institute of Science, Rehovot 76100, Israel

2 Department of Physics, Nuclear Research Center Negev, P.O.B. 9001, Beer-Sheva 84015, Israel

3 Department of Mechanical Engineering, Ben-Gurion University of the Negev, Be'er-Sheva, Israel
}

independent of any characteristic length or time scales. Using dimensional arguments it is possible to show that if the flow is determined by a set of constants, using which it is impossible to construct a constant with the dimensions of length or time, then the flow fields $f(r, t)$ must be of the form $f(r, t)=t^{\alpha_{f}} F(r / R)$ with $R \propto( \pm t)^{\alpha}$ (see chapter XII of Zel'Dovich \& Raizer 1967). We show in $\S 2$ that requiring the asymptotic flow to be independent only of characteristic length scales is sufficient for showing, based on dimensional arguments, that the flow must be self-similar. The less restrictive requirement allows a more general form of self-similar solutions, $f(r, t)=R^{\delta_{f}} F(r / R)$ with $\dot{R} \propto R^{\delta}$, which includes the exponential $(\delta=1)$ solutions, $R \propto e^{t / \tau}$. The existence of exponential self-similar solutions has been noted by several authors (e.g. Stanyukovich 1960). However, it was generally assumed that asymptotic solutions, which are of interest, are of a power law form.

In $\S 3$ we show that the asymptotic self-similar solutions describing the propagation of accelerating blast waves, propagating in a cold gas sphere of initial density $\rho \propto r^{-\omega}$ with $\omega>3$, are of the more general form, $\dot{R} \propto R^{\delta}$, with exponential solutions obtained at $\omega=\omega_{c}(\gamma)$, where $\gamma$ is the adiabatic index of the gas. The new solutions obtained here for $\omega \geq \omega_{c}$ extend the family of second type solutions describing the asymptotic flow of accelerating blast waves, which was derived by Waxman \& Shvarts (1993) and was limited to $\omega<\omega_{c}$, to $\omega \geq \omega_{c}$. The properties of the new solutions are analyzed in $\S 3.2$, and self similar solutions describing the $\omega>\omega_{c}$ flow at times later than the finite divergence time are derived in $\S 3.3$. Our results are summarized in $\S$.

It should be noted here that the self-similar solutions derived by Waxman \& Shvarts (1993) exist only for $\omega>\omega_{g}(\gamma)>3$, where $\omega_{g}=3.26$ for $\gamma=5 / 3$ and 
approaches 3 for $\gamma \rightarrow 1$, while the Sedov-von NeumannTaylor solutions provide the correct asymptotic solutions only for $\omega<3$. The asymptotic behavior within the (narrow) range of $3<\omega<\omega_{g}(\gamma)$ is not described by either of the two types of solutions. The nature of the asymptotic flow in this regime is discussed in Gruzinov (2003); Kushnir \& Waxman (2010).

\section{THE GENERAL FORM OF ASYMPTOTIC SELF-SIMILAR SOLUTIONS}

The equations describing adiabatic one-dimensional flow of an ideal gas are (e.g. Zel'Dovich \& Raizer 1967)

$$
\begin{aligned}
\left(\partial_{t}+u \partial_{r}\right) \ln \rho+r^{-(\nu-1)} \partial_{r}\left(r^{\nu-1} u\right) & =0, \\
\left(\partial_{t}+u \partial_{r}\right) u+\rho^{-1} \partial_{r}\left(\gamma^{-1} \rho c^{2}\right) & =0, \\
\left(\partial_{t}+u \partial_{r}\right)\left(c^{2} \rho^{1-\gamma}\right) & =0,
\end{aligned}
$$

where $u, c$, and $\rho$ are the fluid velocity, sound speed and density respectively (the pressure is given by $p=\rho c^{2} / \gamma$ ), and $\nu=1,2,3$ for planar, cylindrical and spherical symmetry respectively.

Consider a solution $f\left(r, t ;\left\{c_{i}\right\}, \gamma\right)$, where $f$ stands for $\rho, u$ or $c$, and $\left\{c_{i}\right\}$ are the set of constants determining the initial and boundary conditions. Let us assume that the flow takes place over a characteristic scale $R(t)$, which diverges or tends to zero monotonically with time (if $R(t)$ is not monotonic then the flow is characterized by some finite length scales corresponding to the extrema of $R$ ). In this case we may replace the variables $\{r, t\}$ with $\{\xi(r, t)=r / R, R\}$, and describe the flow by $\tilde{f}\left(\xi, R ;\left\{c_{i}\right\}, \gamma\right)$, where $f\left(r, t ;\left\{c_{i}\right\}, \gamma\right)=$ $\tilde{f}\left[\xi(r, t), R(t) ;\left\{c_{i}\right\}, \gamma\right]$, and by $\dot{R}\left(R ;\left\{c_{i}\right\}, \gamma\right)$, which determines the relation between $R$ and $t$.

Let us assume next that in the limit $R \rightarrow \infty(0)$ the flow becomes independent of any characteristic length scales. That is, that in the limit $R \rightarrow \infty(0)$ the flow is determined by only a subset of $\left\{c_{i}\right\}$, denoted $\left\{b_{i}\right\}$, from which a constant with the dimensions of length cannot be constructed. The dimensions of each parameter $b_{k}$ may be considered as a three dimensional vector $\vec{v}_{k},\left[b_{k}\right]=M^{v_{1}} L^{v_{2}} T^{v_{3}}$, where $M, L$, and $T$ are the units of mass, length, and time, respectively. Since a constant with the dimensions of length may not be constructed using $\left\{b_{i}\right\}$, there are at most two constants with independent dimension vectors. As we shall see below, there must exit two constants with independent dimensions. Denoting these by $\left\{a_{1}, a_{2}\right\}$, and expressing the other constants as $b_{i}=\gamma_{i} a_{1}^{v_{i 1}} a_{2}^{v_{i 2}}$ with dimensionless $\gamma_{i}$, the asymptotic solution may be written as $\tilde{f}_{\text {as. }}\left(\xi, R ; a_{1}, a_{2},\left\{\gamma_{i}\right\}, \gamma\right), \dot{R}_{\text {as. }}\left(R ; a_{1}, a_{2},\left\{\gamma_{i}\right\}, \gamma\right)$. Since the dimensions of $\left\{R, a_{1}, a_{2}\right\}$ are independent (otherwise it would be possible to construct a constant with dimensions of length using $\left.\left\{a_{1}, a_{2}\right\}\right)$, there is a single combination (product of powers) of $\left\{R, a_{1}, a_{2}\right\}$ that has a given dimension vector. This implies that $\tilde{f}_{\text {as. }}$ must be of the form $\tilde{f}_{\text {as. }}=a_{1}^{\alpha_{f 1}} a_{2}^{\alpha_{f 2}} R^{\alpha_{f}} F\left(\xi ;\left\{\gamma_{i}\right\}, \gamma\right)$ and $\dot{R}_{\text {as. }}$ must be of the form $\dot{R}_{\text {as. }}=a_{1}^{\delta_{1}} a_{2}^{\delta_{2}} R^{\delta} F_{R}\left(\left\{\gamma_{i}\right\}, \gamma\right)$.

Thus, the assumption that the asymptotic solution is independent of any characteristic length scales implies that it must be a self-similar solution of the form

$$
u=\dot{R} \xi U(\xi), \quad c=\dot{R} \xi C(\xi), \quad \rho=B R^{\epsilon} G(\xi)
$$

with

$$
\dot{R}=A R^{\delta}
$$

The spatial part of $u(c)$ was chosen as $\xi U(\xi)(\xi C(\xi))$ for convenience (see below). Note, that the dimensions of $A$ and $B$ are independent. These solutions include the $R \propto( \pm t)^{\alpha}$ solutions, obtained for $\delta=(\alpha-1) / \alpha \neq 1$, and the exponential $R \propto e^{t / \tau}$ solutions obtained for $\delta=1$.

Substituting the ansatz of eqs. (2) and (3) in the hydrodynamic equations (eq. (1)), the partial differential equations are replaced with a single ordinary differential equation,

$$
\frac{d U}{d C}=\frac{\Delta_{1}(U, C)}{\Delta_{2}(U, C)}
$$

and one quadrature

$$
\frac{d \ln \xi}{d U}=\frac{\Delta(U, C)}{\Delta_{1}(U, C)} \quad \text { or } \quad \frac{d \ln \xi}{d C}=\frac{\Delta(U, C)}{\Delta_{2}(U, C)} .
$$

$G$ is given implicitly by

$$
(\xi C)^{-2(\nu+\epsilon)}|1-U|^{\lambda} G^{(\gamma-1)(\nu+\epsilon)+\lambda} \xi^{\nu \lambda}=\text { Const. },
$$

with

$$
\lambda=-(\gamma-1) \epsilon+2 \delta .
$$

The functions $\Delta, \Delta_{1}$, and $\Delta_{2}$ are

$$
\begin{aligned}
\Delta & =C^{2}-(1-U)^{2}, \\
\Delta_{1} & =U(1-U)(1-U-\delta)-C^{2}\left(\nu U+\frac{\epsilon+2 \delta}{\gamma}\right), \\
\Delta_{2} & =C\{(1-U)(1-U-\delta) \\
& -\frac{\gamma-1}{2} U[(\nu-1)(1-U)+\delta]-C^{2} \\
& \left.+\frac{2 \delta-(\gamma-1) \epsilon}{2 \gamma} \frac{C^{2}}{1-U}\right\} .
\end{aligned}
$$

\section{ACCELERATING BLAST WAVE SOLUTIONS}

Consider the blast wave produced by the deposition of energy $E$ within a region of characteristic size $d$ at the center of an initially cold $(p=0$ at $r>d)$ gas sphere with initial density $\rho_{0}=K r^{-\omega}($ at $r>d)$. As the shock radius $R$ diverges, it is reasonable to assume that the flow becomes independent of any length scale determined by the initial conditions, i.e. that $R(t)$ is the only relevant length scale. In this case, the flow should approach a self-similar solution of the form given by eqs. (2) and (3). Since the density just behind the shock wave is, for strong shocks, a constant factor, $(\gamma+1) /(\gamma-1)$, times the density just ahead of the shock, we must have $\epsilon=-\omega$, and we may choose $B=K$. With this normalization, the Rankine-Hugoniot relations at the shock front determine the boundary conditions for the self-similar solutions to be (e.g. Zel'Dovich \& Raizer 1967)

$$
U(1)=\frac{2}{\gamma+1}, \quad C(1)=\frac{\sqrt{2 \gamma(\gamma-1)}}{\gamma+1}, \quad G(1)=\frac{\gamma+1}{\gamma-1} .
$$

The only parameter of the self-similar solution that remains to be determined is $\delta$. The methods described below for determining $\delta$, and for analyzing the resulting solutions' properties, are similar to those described in (Waxman \& Shvarts 1993). The discussion in the following subsections is therefore very concise. The reader may refer to Waxman \& Shvarts (1993) for more elaborate explanations. 


\section{1. $\omega<\omega_{c}$}

In the Sedov-von Neumann-Taylor analysis it is assumed that the second dimensional constant, in addition to $K$, that determines the self-similar solution is $E$. In this case dimensional considerations imply $R \propto\left(E t^{2} / K\right)^{1 /(5-\omega)}$, i.e. $\delta=(\omega-3) / 2$. As explained in detail in (Waxman \& Shvarts 1993), the Sedov-von Neumann-Taylor solutions are the correct asymptotic solutions only for $\omega<3$, for which $\delta<0$ and the blast wave decelerates with time. For larger values of $\omega$ the mass and energy contained within the self-similar solution are infinite, reflecting the fact that the initial gas mass at $r>d$ diverges for $d \rightarrow 0$. It was therefore suggested by Waxman \& Shvarts (1993) that for $\omega>3$ the asymptotic solution is given by a self-similar solution only over part of the $\{\xi, R\}$ plane, bounded by $\xi=1$ and $\xi_{c}(R)<1$, and by a different solution at $0<\xi<\xi_{c}(R)$.

Since $\xi_{c}(R)$ must be a contact or a weak discontinuity, $\xi_{c}(R)$ must be a characteristic of the self-similar solution. For the self-similar flow, the characteristic lines

$$
C_{0}: \frac{d r_{0}}{d t}=u, \quad C_{ \pm}: \frac{d r_{ \pm}}{d t}=u \pm c
$$

are given by

$$
\begin{aligned}
C_{0} & : \frac{d \ln \xi_{0}}{d \ln R}=U\left(\xi_{0}\right)-1, \\
C_{ \pm} & : \frac{d \ln \xi_{ \pm}}{d \ln R}=U\left(\xi_{ \pm}\right) \pm C\left(\xi_{ \pm}\right)-1 .
\end{aligned}
$$

The flow just behind the shock is always subsonic: the shock-front point $\{U=U(1), C=C(1)\}$ lies above the "sonic-line" $U+C=1$, which implies that $C_{+}$characteristics emerging from points just behind the shock always overtake it. $C_{+}$characteristics that do not overtake the shock exit only if the self-similar solution crosses the $U+C=1$ line in the $\{U, C\}$ plane into the region where $U+C<1$. Requiring $\xi_{c}$ to coincide with a $C_{+}$ characteristic, that does not overtake the shock, implies therefore that the solution must cross the $U+C=1$ line. Since $\Delta=0$ along the sonic line, eqs. (5) imply that a physical solution must cross the sonic line at a singular point $\Delta_{1}=\Delta_{2}=0$ (otherwise $U(\xi)$ or $C(\xi)$ are not single valued). This requirement determines the correct value of $\delta$ for the $\omega>3$ asymptotic solutions.

The self-similar solutions obtained in this way for $\omega>$ 3 were analyzed in detail in (Waxman \& Shvarts 1993). They describe accelerating blast waves, with $\delta>0$, and approach the singular point $\{U=1-\delta, C=0\}$ as $\xi \rightarrow 0$. Analyzing the behavior of the solutions near this singular point, it was shown that although the mass and energy contained in the self-similar solution are infinite, the mass and energy contained within the region $\xi_{c}(R)<\xi<1$, where $\xi_{c}(R)$ is a $C_{+}$characteristic which satisfies $\xi_{c}(R) \rightarrow 0$ as $R \rightarrow \infty$, approach finite values as $R \rightarrow \infty$. Moreover, it was shown that $\xi_{c}(R) R \propto t$ as $R \rightarrow \infty$, implying that the asymptotic flow within the region $0<r<\xi_{c}(R) R$ is described by the self-similar solution of expansion into vacuum. Finally, it was demonstrated in (Waxman \& Shvarts 1993) by numerical simulations that the asymptotic behavior described above is indeed approached for $R / d \gg 1$.

$$
\text { 3.2. } \omega \geq \omega_{c}
$$

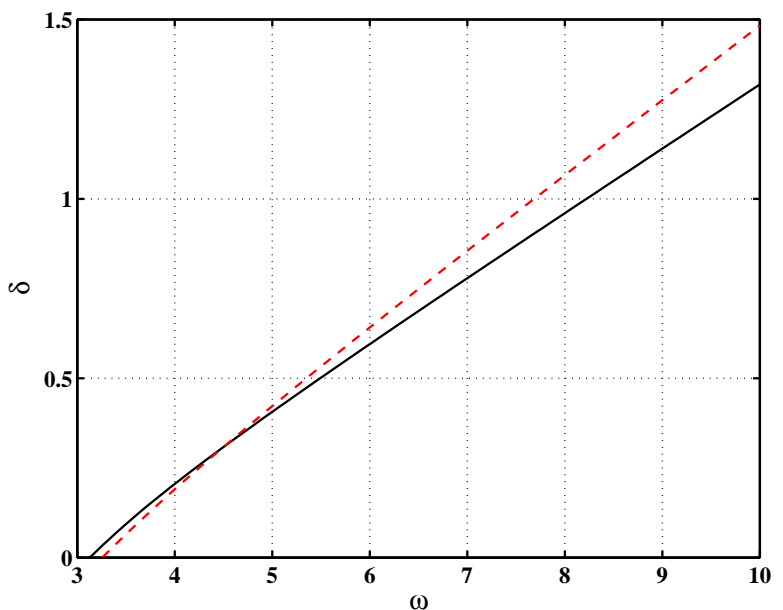

FIG. $1 .-\delta(\omega)$ for $\gamma=5 / 3$ (red dashed line) and $\gamma=4 / 3$ (solid black line) determined by the requirement that the $C(U)$ curve crosses the sonic line at a singular point. As noted in (Waxman \& Shvarts 1993), there are no solutions satisfying this requirement within some narrow range $3<\omega<\omega_{g}(\gamma)\left(\omega_{g}=3.26\right.$ for $\gamma=5 / 3$ and approaches 3 for $\gamma \rightarrow 1)$. The nature of the asymptotic flow in this regime is discussed in Kushnir \& Waxman 2010).

In the analysis of (Waxman \& Shvarts 1993) it was assumed that the asymptotic solutions are of the form $R \propto t^{\alpha}$. It was found that $\alpha$ diverges as $\omega$ approaches a finite value, $\omega_{c}(\gamma)\left(\omega_{c} \sim 8\right.$ for $4 / 3<\gamma<5 / 3$ and diverges for $\gamma \rightarrow 1$, see fig. 5 of Waxman \& Shvarts 1993). Allowing solutions of the more general form, $\dot{R} \propto R^{\delta}$ as suggested in $\S 2$, the divergence of $\alpha$ suggests that the asymptotic solution for $\omega=\omega_{c}(\gamma)$ is an exponential solution, $R \propto e^{t / \tau}$ (recall that $\delta=(\alpha-1) / \alpha$ ). Searching for solutions of the form $\dot{R} \propto R^{\delta}$, that cross the sonic line at a singular point, we find that such solutions do exist for $\omega \geq \omega_{c}$. $\delta(\omega)$ is shown for $\gamma=5 / 3$ and $\gamma=4 / 3$ in figure 1. We find $\delta=1$ for $\omega=\omega_{c}(\gamma)$, implying exponential expansion, and $\delta>1$ for $\omega>\omega_{c}(\gamma)$, implying that the shock radius diverges in finite time, $R \propto(-t)^{1 /(1-\delta)}$. Fig. 2 presents a comparison of the $C(U)$ curves obtained by numerically solving the hydrodynamic equations, eq. (1), with those obtained by solving the self-similar eq. (4). The numerical results suggest that the self-similar solutions provide a correct description of the asymptotic flow.

For $\delta \geq 1$, the self-similar solution approaches the singular point $\{U=0, C=0\}$ as $\xi \rightarrow 0$. Solving the self-similar equations in the vicinity of this point we find that in the limit of $\xi \rightarrow 0$ the solutions are given by

$$
U=-\frac{1}{\ln \xi}, \quad C \propto U^{(3 \gamma-1) / 2}, \quad G \propto \xi^{-\omega}(\ln \xi)^{\omega-3}
$$

for $\delta=1$, and by

$$
U=U_{1} \xi^{\delta-1}, \quad C=C_{1} \xi^{\delta-1}, \quad G=G_{1} \xi^{-\omega},
$$

where $U_{1}, C_{1}, G_{1}$ are constants, for $\delta>1$. Using eqs. (12) and (13) we find that the $C_{+}$characteristics originating from points below the sonic line are given in the limit $R \rightarrow \infty$ by

$$
\xi_{+} R \propto \begin{cases}\left|\ln \xi_{+}\right|, & \delta=1 \\ \text { Const. } & \delta>1\end{cases}
$$




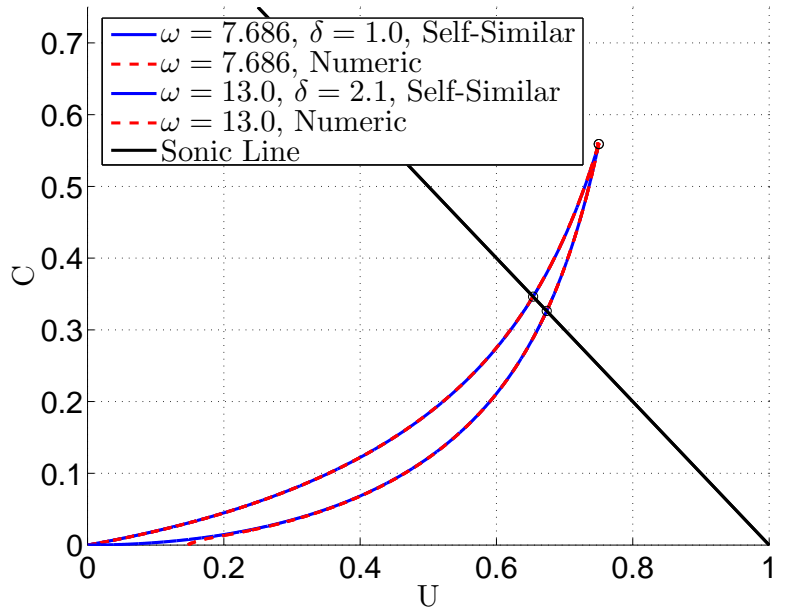

FIG. 2.- A comparison of the $C(U)$ curves obtained from numerical solutions of the hydrodynamic equations, eq. (1), with those obtained by solving the self-similar eq. (4). Results are shown for $\gamma=5 / 3$ and two values of $\omega, \omega=7.686$ for which the self-similar analysis yields $\delta=1.0$ (lower curve), and $\omega=13.0$, for which the self-similar analysis gives $\delta=2.10$. Numerical solutions are shown for $R / d=500$ (where $R$ is the shock radius and $d$ the radius of the sphere within which the energy is initially deposited).

The energy contained in a region of the self-similar solution, bounded by $\xi=1$ and a $C_{+}$characteristic $\xi_{+}(R)$, is given by

$$
\begin{aligned}
E_{+}(R)= & 4 \pi K A^{2} R^{3-\omega+2 \delta} \\
& \times \int_{\xi_{+}(R)}^{1} d \xi \xi^{4} G\left[\frac{1}{2} U^{2}+\frac{1}{\gamma(\gamma-1)} C^{2}\right] .
\end{aligned}
$$

In order for the energy $E_{+}$not to diverge as $R \rightarrow \infty$ and $\xi_{+}(R) \rightarrow 0$, we must have

$$
\delta<\frac{\omega-3}{2}
$$

This is satisfied for the solutions presented in fig. 1] For $\delta$ values satisfying eq. (16), the integral over $\xi$ diverges as $\xi_{+}(R) \rightarrow 0$, while $R^{3-\omega+2 \delta}$ vanishes as $R \rightarrow \infty$. Using eqs. (12)-(14), it is straight forward to verify that $E_{+}(R) \rightarrow$ Const. as $R \rightarrow \infty$. Thus, the energy contained in the region of the flow which is described by the self-similar solution, $\xi_{c}(R)<\xi<1$, approaches a finite value as $R \rightarrow \infty$. A similar calculation shows that the mass contained in this region also approaches a finite value.

$$
\text { 3.3. } t>0
$$

For $\delta>1$, the shock radius diverges at a finite time, $t=$ 0 . This implies that the spatial distribution of the flow fields at the divergence time is described by the $\xi \rightarrow 0$ behavior of the solution, which is given by eq. (13). That is, at $t=0$ the spatial distribution of the flow fields is

$$
u \propto r^{\delta}, \quad c \propto u, \quad \rho \propto r^{-\omega}
$$

(Note, that this form of the flow fields applies for $r>r_{0}$ where $r_{0}$ is the finite radius approached by $\xi_{c} R$ as $R$ diverges, see eq. (14)). Since the distribution of flow fields at $t=0$ has no characteristic scale, the flow at $t>0$ will be described by a self-similar solution.
The $t>0$ self-similar solution may be constructed as follows. Denoting the scaling radius of this solution by $\tilde{R}$, we choose

$$
\dot{\tilde{R}}=-A \tilde{R}^{\delta}
$$

with the same values of $A$ and $\delta$ obtained for the $t<0$ solution. This implies $\left.\tilde{R} \propto t^{1 /(1-\delta}\right)$, i.e. $\tilde{R}$ is decreasing with $t$ and diverges as $t \rightarrow 0_{+}$. Next, we choose a $C(U)$ curve that approaches in the limit $\tilde{\xi} \rightarrow 0$, where $\tilde{\xi} \equiv r / \tilde{R}$, the same singular point approached in this limit by the $t<0$ solution, $U=C=0$ :

$$
U=-U_{1} \tilde{\xi}^{\delta-1}, \quad C=C_{1} \tilde{\xi}^{\delta-1}, \quad G=G_{1} \tilde{\xi}^{-\omega}
$$

with the same values of $U_{1}, C_{1}, G_{1}$ obtained for the $t<0$ solution (Note, that for $\delta>1$ any straight line $C \propto U$ is a solution of eq. (4) near $U=C=0$ ). It is straight forward to verify that the $t>0$ self-similar flow thus defined approaches the form given by eq. (17) as $t \rightarrow 0_{+}$.

In the limit $t \rightarrow \infty, \tilde{R} \rightarrow 0$ and the spatial distribution of the flow fields is described by the $\tilde{\xi} \rightarrow \infty$ behavior of the solution. In the limit $\tilde{\xi} \rightarrow \infty$, the $C(U)$ curve of the $t>0$ solution approaches the singular point $\{U=1-\delta, C=0\}$. The behavior of the self-similar solutions in the vicinity of this point was analyzed in (Waxman \& Shvarts 1993).

\section{SUMMARY}

We have discussed the asymptotic behavior of adiabatic one dimensional flows of an ideal gas, which take place over a characteristic scale $R$ that diverges or tends to zero. We have shown in $\S 2$ that requiring the asymptotic flow to be independent of characteristic length scales implies, based on dimensional arguments, that the flow must be self-similar, with flow fields given by eq. (2) and $R$ satisfying eq. (3), $\dot{R} \propto R^{\delta}$. The ordinary differential equations determining the self-similar profiles are given by eqs. (4)-(8).

In $\S 3$ we have shown that the asymptotic self-similar solutions describing the propagation of accelerating blast waves, propagating in a cold gas sphere of initial density $\rho \propto r^{-\omega}$ with $\omega>3$, are of the general form $\dot{R} \propto R^{\delta}$, with exponential solutions obtained at $\omega=\omega_{c}(\gamma) . \delta(\omega, \gamma)$ is shown in fig. (1) for $\gamma=4 / 3,5 / 3$. Fig. 2 demonstrates that numerical solutions of the hydrodynamic equations, eq. (1), indeed approach these self-similar solutions as $R$ diverges. The properties of the self-similar solutions obtained for $\omega \geq \omega_{c}(\gamma)$ are analyzed in $\S 3.2$ The $C(U)$ curves of these solutions approach the singular point $\{U=0, C=0\}$ as $\xi \rightarrow 0$. Analyzing the behavior of the solutions near this singular point we have shown that the energy and mass contained in region of the $\{\xi, R\}$ plane bounded by $\xi=1$ and $\xi=\xi_{+}(R)$, where $\xi_{+}(R)$ is a $C_{+}$ characteristic that approaches $\xi=0$ as $R$ diverges, both tend to finite constants as $R$ diverges.

For $\delta>1$, the shock radius diverges at a finite time, $t=0$. This implies that the spatial distribution of the flow fields at the divergence time is described by the $\xi \rightarrow$ 0 behavior of the solution, which is given by eq. (13). This implies that at $t=0$ the spatial distribution of the flow fields is given by eq. (17). In $\S 3.3$ we have shown that the $t>0$ flow is described in this case by a selfsimilar solution with the same value of $\delta$ as that of the 
$t<0$ solution, see eq. (18), and spatial profiles determine by eq. (19). calculations presented in figure 2. This research was supported in part by ISF, AEC, and Minerva grants.

We thank D. Kushnir for carrying out the numerical

\section{REFERENCES}

Barenblatt, G. I. 1996, Scaling, Self-similarity, and Intermediate Asymptotics (Cambridge: Cambridge University Press)

Gandel'Man, G. M. \& Frank-Kamenetskii, D. A. 1956, Soviet Physics Doklady, 1, 223

Gruzinov, A. 2003, arXiv:astro-ph/0303242

Kushnir, D. \& Waxman, E. 2010, arXiv:1002.3869

Matzner, C. D. \& McKee, C. F. 1999, ApJ, 510, 379

Ostriker, J. P. \& McKee, C. F. 1988, Reviews of Modern Physics, 60,1

Sakurai, A. 1960, Communications on Pure and Applied Mathematics, 13, 353

Sedov, L. I. 1946, Prikl. Mat. Mekh., 10, 241

- 1959, Similarity and Dimensional Methods in Mechanics (New York: Academic Press)
Stanyukovich, K. P. 1960, Unsteady Motion of Continuous Media, ed. M. Holt (New York: Academic Press)

Taylor, G. 1950, Royal Society of London Proceedings Series A, 201, 159

Von Neumann, J. 1947, Blast Waves, Los alamos sci. lab. tech. series, vol. 7, Los Alamos, NM

Waxman, E., Mészáros, P., \& Campana, S. 2007, ApJ, 667, 351

Waxman, E. \& Shvarts, D. 1993, Physics of Fluids, 5, 1035

Zel'Dovich, Y. B. \& Raizer, Y. P. 1967, Physics of shock waves and high-temperature hydrodynamic phenomena (New York: Academic Press) 